BULLETIN (New Series) OF THE

AMERICAN MATHEMATICAL SOCIETY

Volume 45, Number 4, October 2008, Pages 485-487

S 0273-0979(08)01230-5

Article electronically published on July 3, 2008

\title{
SOME COMMENTARY ON ATLE SELBERG'S MATHEMATICS
}

\author{
D. HEJHAL AND P. SARNAK
}

One way of characterizing Atle Selberg's mathematical genius is that he had a "golden touch". In those domains he thought about in depth, he saw further than generations before him, repeatedly uncovering truths lying below the surface. His breakthroughs on long-standing problems were based on imaginative and novel ideas which, once digested, were appreciated as simple and decisive.

The impact of his work is far greater than what appears in his collected works $[\mathrm{S}]$. He developed many tools and techniques which are the basis of far-reaching achievements by others in contiguous fields. There are few to whom the term "mathematician's mathematician" applies so well.

It is impossible in a couple of paragraphs to do justice to Selberg's achievements and his impact. Our hope is that by pointing to some of his major results and placing them in a modern context, we can at least give the reader a taste.

Selberg burst into the limelight with his proof that the Riemann zeta-function has a positive proportion of its zeros on the critical line. More important than the result was the technique of "mollification" (as it is referred to today) that he introduced in the proof. The method of weighting averages involving zeta and $L$-functions by squares of (Dirichlet) polynomials whose coefficients are optimized only at the end of the analysis allowed him to smooth out the large values of zeta on the critical line. This, in turn, enabled him to examine the zeta-function on short intervals and to establish the positive proportion theorem.

The mollification method and its many variants remains today as one of the most powerful tools in the study of more general zeta and $L$-functions on the critical line. Selberg himself, at the age of 80 , in a technical tour-de-force, extended these ideas to show that any real linear combination of modular $L$-functions of a certain type also has a positive proportion (though certainly not all!) of its zeros on the line $\operatorname{Re}(s)=1 / 2$.

Selberg's original work from the early 1940's on the zeta-function led him to his elegant and powerful "lambda-squared" sieve and, from there, to an in-depth analysis of sieve methods and especially their limits. In particular, he identified and clarified various fundamental issues intrinsically associated with sieve methods, such as the parity problem. Selberg's "fundamental formula", which lies at the heart of the celebrated elementary proof of the prime number theorem, also arose naturally from this work on the zeta-function and the sieve.

During this same period (1940-1950), Selberg developed what is known today as the Rankin-Selberg method, as well as the Selberg Integral. The former was

Received by the editors June 27, 2008.

2000 Mathematics Subject Classification. Primary 01A60, 01A65.

(C)2008 American Mathematical Society 
discovered independently by Rankin and Selberg, and furnishes the first $L$-function beyond that of Hecke that can be associated to a modular form. Both Rankin and Selberg made use of this $L$-function in giving nontrivial bounds toward Ramanujan's well-known conjecture concerning the Fourier coefficients $\tau(n)$ of $\Delta$ (the discriminant of an elliptic curve). Interestingly, their method and its generalizations were one of the new ingredients beyond Grothendieck's work that Deligne introduced in the function-field setting in his proof of the Weil Conjectures and - as a consequence-Ramanujan's Conjecture.

The Selberg Integral is a multivariable generalization of Euler's integral for the beta function. It is remarkable that such a result was only discovered in the 1940's, and interesting that this paper by Selberg (one of his few in Norwegian) went largely unnoticed until 1979, since related special cases of this integral were being formulated as conjectures up until that time (see the article by Forrester and Warnaar in this issue). Being an elementary and basic result in calculus, this integral has many applications ranging from mathematics and statistics to statistical physics and engineering.

Beginning around 1950, Selberg turned his attention to modular and more general automorphic forms. His development of the trace formula - as well as the crucial accompanying theory giving the meromorphic continuation of Eisenstein series - mark the beginning of the modern theory. One could argue that the trace formula was to some extent already in the air; in fact, some special cases of the trace formula were discovered independently by Eichler during approximately the same time period. However, in the case of the Eisenstein series (and related instances of the trace formula), Selberg arrived on completely virgin territory and needed to lay the foundations from scratch.

In its simplest setting, the trace formula relates the eigenvalues of the Laplacian to the lengths of the closed geodesics on a hyperbolic surface. Selberg used it to investigate the finer structure of these quantities and introduced a zeta-like function associated with the closed geodesics which is known today as the Selberg Zeta Function. Particularly striking in this context is the fact that, not only is there an analog of the prime number theorem for the geodesics, but that it is obtainable with an error term roughly comparable in size and format to what would hold for the ordinary primes under the assumption of the Riemann Hypothesis.

Fundamental work of Langlands established the meromorphic continuation of Eisenstein series for a general reductive group and this led him to what has become one of the holy grails of the subject: the Principle of Functoriality. The theory of Eisenstein series, combined with the general noncompact adelic trace formula developed by Arthur over many years, is among the most powerful tools that we have today in the theory of automorphic forms. A list of well-known results whose proof relies on the trace formula would cover many pages.

Selberg was the first to realize (in 1963) that the classical Ramanujan-Petersson Conjecture concerning the size of Fourier coefficients of holomorphic cusp forms has an archimedean analog for the case of more general Maass forms (instead of being holomorphic, such forms are eigenfunctions of corresponding differential operators). He formulated the basic conjecture for these, which today is known as "Selberg's Eigenvalue Conjecture". He also provided techniques to prove approximations towards such conjectures (even over more general Lie groups) and pointed to potentially far-reaching applications in various analytic problems in number theory. The 
representation-theoretic formulations and generalizations of these conjectures are fundamental ones in the modern theory of automorphic forms, and have wide - and often unexpected - applications.

Selberg's work in automorphic forms and number theory led him naturally to the study of lattices (that is, discrete subgroups of finite covolume) in semi-simple Lie groups. His proof of local rigidity and, as a consequence, algebraicity of the matrix entries of cocompact lattices in groups $\operatorname{such}$ as $\operatorname{SL}(n, \mathbb{R}), n>2$, marked the beginnings of modern rigidity theory. His results were followed by proofs of local rigidity for cocompact lattices in all groups other than the familiar $\operatorname{SL}(2, \mathbb{R})$, where its failure reflects the well-known local deformation theory of Riemann surfaces. These results inspired Mostow to find and prove his celebrated "strong rigidity" results for such lattices in groups other than $\mathrm{SL}(2, \mathbb{R})$.

From his work on local rigidity and algebraicity, Selberg was led to the bold conjecture that, in the higher rank situation, much more is true; namely, that all lattices are arithmetic (i.e., they can be constructed by some general arithmetic means). He was able to prove this conjecture in the simplest case of a non-cocompact irreducible lattice in the product of at least two $\operatorname{SL}(2, \mathbb{R})$ 's. The full Selberg arithmeticity conjecture in groups of rank at least two was established by Margulis, who introduced measure- and p-adic theoretic ideas into the problem, as well as what is now called "super-rigidity".

Even with his uncanny intuition about this problem, Selberg could not have predicted what should happen in certain rank one spaces, such as quaternionic hyperbolic space and the Cayley plane. Corlette's unexpected proof using harmonic maps of archimedean super-rigidity for lattices in these groups was followed by a flurry of works which, among other things, led to proofs of arithmeticity in these cases - as well as new proofs of arithmeticity in the higher rank cases.

From our brief description, it should be evident that Selberg's work has an ongoing and rich vitality. There is no doubt that Selberg thought in depth about some notoriously difficult problems such as the Riemann Hypothesis, but his approach was pragmatic and realistic; and, in thinking about and around such problems, he uncovered techniques and gems which are of equal importance and which have a permanent place in mathematics.

\section{REFERENCES}

[S] A. Selberg, Collected Papers, vols. 1 and 2, Springer-Verlag, 1989 and 1991.

School of Mathematics, University of Minnesota, Minneapolis, Minnesota 55455, and Department of Mathematics, Uppsala University, S-75106 Uppsala, Sweden

E-mail address: hejhal@math.uu.se

School of Mathematics, Institute for Advanced Study, Princeton, New Jersey 08540, and Department of Mathematics, Princeton University, Princeton, New Jersey 08544

E-mail address: sarnak@math.princeton.edu 